\title{
Statistical separation strategy to analyze velocity structure obtained by seismic tomography
}

\author{
Wolfgang Weinzierl ${ }^{1}$ and Heidrun Kopp ${ }^{1}$
}

\begin{abstract}
General solutions of inverse problems often can be obtained by introducing probability distributions to sample the model space. We have developed a simple approach to define an a priori space in a tomographic study and retrieve the velocity/depth posterior distribution by a Monte Carlo method. Utilizing a fitting routine designed for very low statistics to set up and analyze the obtained tomography results, we can statistically separate the velocity/depth model space derived from inverting seismic refraction data. A profile acquired in the Lesser Antilles subduction zone reveals the effectiveness of this approach. Resolution analysis of the structural heterogeneity includes a divergence analysis that can dissect long, wide-angle profiles for deep crust and upper mantle studies. The complete information of any parameterized physical system is contained in the a posteriori distribution. Methods for analyzing and displaying key properties of the a posteriori distributions of highly nonlinear inverse problems are therefore essential in the scope of any interpretation. It is possible to map velocity variations in their extent and structure by measuring the total as well as relative divergence of the velocity structure in the a posteriori space. We have applied the divergence analysis to a part of the transect where a backstop structure has been identified, and the method resolves shallow features and returns information concerning the confidence level of results. Assuming a relationship between forearc and backstop, we can obtain a structural image in accordance with previous interpretations.
\end{abstract}

\section{INTRODUCTION}

Numerous studies dealing with inverse problems have been approached in as many ways as there are questions to be answered by them (Parker, 1994). The theory of seeking parameters by indirect measurements has been applied successfully by Parker and
Dziewonski (1995) in a more phenomenological fashion than the one Hjelt (1992) chooses by inference in a more qualitative way or the rigorous mathematical approach of Kirsch (1996). The reformulation of the theory in a nonparametric fashion has enabled a statistical approach and error analysis to quantitatively evaluate and investigate the solutions to any inverse problem.

In the Bayesian formulation, the most general solution of any inverse problem is a probability distribution of the model space. Analytic techniques solving this problem only apply in the simplest case, i.e., only one global minimum and no local minima exist. Because this ideal case is almost never met, the model space must be explored extensively. There are numerous examples of solutions to inverse problems by means of Monte Carlo methods (e.g., Press, 1968, 1971; Mosegaard and Tarantola, 1995; Jacobsen et al., 1996; Mosegaard and Sambridge, 2002; Sambridge and Mosegaard, 2002).

A major concern in the Bayesian approach is knowing the a priori distribution for setting up the starting models that sample the model space (Hansen et al., 2006). One way of simplifying and reducing such a search to a limited band of models for inversion is to introduce envelopes for parameter estimates. A difficulty with this approach is inconclusive knowledge of sampling the complete model space because we are introducing boundary conditions on the P-wave velocities at depth (Sato and Kennett, 2000). By expanding or broadening the envelopes, we can expand the a priori distributions and decrease the effects of the boundary conditions.

We present a statistical separation strategy to explore the velocity/ depth model space derived from inverting seismic refraction data. After an initial tomography with a simple 1D velocity setup hanging below the seafloor, we separate the transect according to its assumed tectonic units, i.e., their velocity/depth distribution. Fitting the velocity/depth distributions $V(z)$ in predefined regions on the grid provides us the essential probability density distributions for setting up a Monte Carlo ensemble for a subsequent inversion. To test our approach, we use a data set from the Lesser Antilles margin south of Guadeloupe. The 280-km-long profile traverses the island arc from the active arc region up to the accretionary prism. We invert more than 22,500 first-arrival traveltimes in over 50 starting models to enhance the statistical resolution of the final average model.

Manuscript received by the Editor 21 July 2009; revised manuscript received 12 January 2010; published online 2 August 2010.

IFM-GEOMAR Geodynamics, Kiel, Germany.E-mail: wweinzierl@ifm-geomar.de; hkopp@ifm-geomar.de.

(C) 2010 Society of Exploration Geophysicists. All rights reserved. 
The advantages of the Monte Carlo method become even more apparent in the subsequent resolution analysis. We use the total as well as relative divergence of the velocity structure of the a posteriori information to map velocity variations in their extent and structure.

\section{DATA ACQUISITION AND TOMOGRAPHIC MODELING}

We inverted first-arrival data from a marine transect acquired with the RV Maria S. Merian, cruise 4, leg 2 (MSM04/2), in 2007 in the Lesser Antilles subduction zone (Figure 1). The profile was shot with a five-element seismic source array, a volume of $112 \mathrm{~L}$, and a trigger interval of $60 \mathrm{~s}$ at a ship speed of $3.7 \mathrm{kn}$ on average, resulting in a shot spacing of approximately $100 \mathrm{~m}$. Forty-four ocean-bottom seismometer (OBS) receivers were positioned at 5-km spacing along the profile. The $280-\mathrm{km}$-long profile traversed the island arc and ended $70 \mathrm{~km}$ southeast of the trench on the accretionary prism. Standard processing of the OBS data included clock-drift correction, relocalization, deconvolution, and filtering. Signal-to-noise ratios varied considerably, and clear arrivals on some stations could be traced to $100-130-\mathrm{km}$ offsets. The transmitted energy decreased rapidly, entering the accretionary complex where first arrivals could be identified for up to $20-\mathrm{km}$ offsets on average.

An initial 2D velocity/depth model along the transect was obtained using the tomographic inversion method of Korenaga et al. (2000). The velocity field $V_{i k}=V\left(x_{i}, z_{k}\right)$ was parameterized by a homogeneous grid of nodes with $1 \leq i \leq n_{x}$ and $1 \leq k \leq n_{z}$ below the seafloor. The matrix equation

$$
\mathbf{d}_{\mathrm{th}}=\mathbf{G} \delta \mathbf{m},
$$

with $\mathbf{d}_{\text {th }}$ the theoretical traveltime residual vector, $\mathbf{G}$ the Frechet derivative matrix, and $\delta \mathbf{m}$ an unknown model perturbation, formed the basis of this traveltime tomography. This linearized inversion procedure was adopted from Toomey et al. (1994) and applied iteratively until the model converged. The models were parameterized with a lateral nodal spacing of $0.5 \mathrm{~km}$ and variable vertical spacing of $0.05 \mathrm{~km}$ within the upper $2 \mathrm{~km}$, increasing to $0.5 \mathrm{~km}$ at $z_{\max }=$ $25 \mathrm{~km}$. To stabilize the inversion, we used depth-dependent smoothing constraints and correlation lengths. Correlation lengths of $1-5 \mathrm{~km}$ horizontally and $0.1-1 \mathrm{~km}$ vertically provided reliable results while using computationally less expensive, larger smoothing constraints.

The starting model for the first tomographic inversion was a simple layered $1 \mathrm{D}$ velocity model. The result of this inversion gave us an

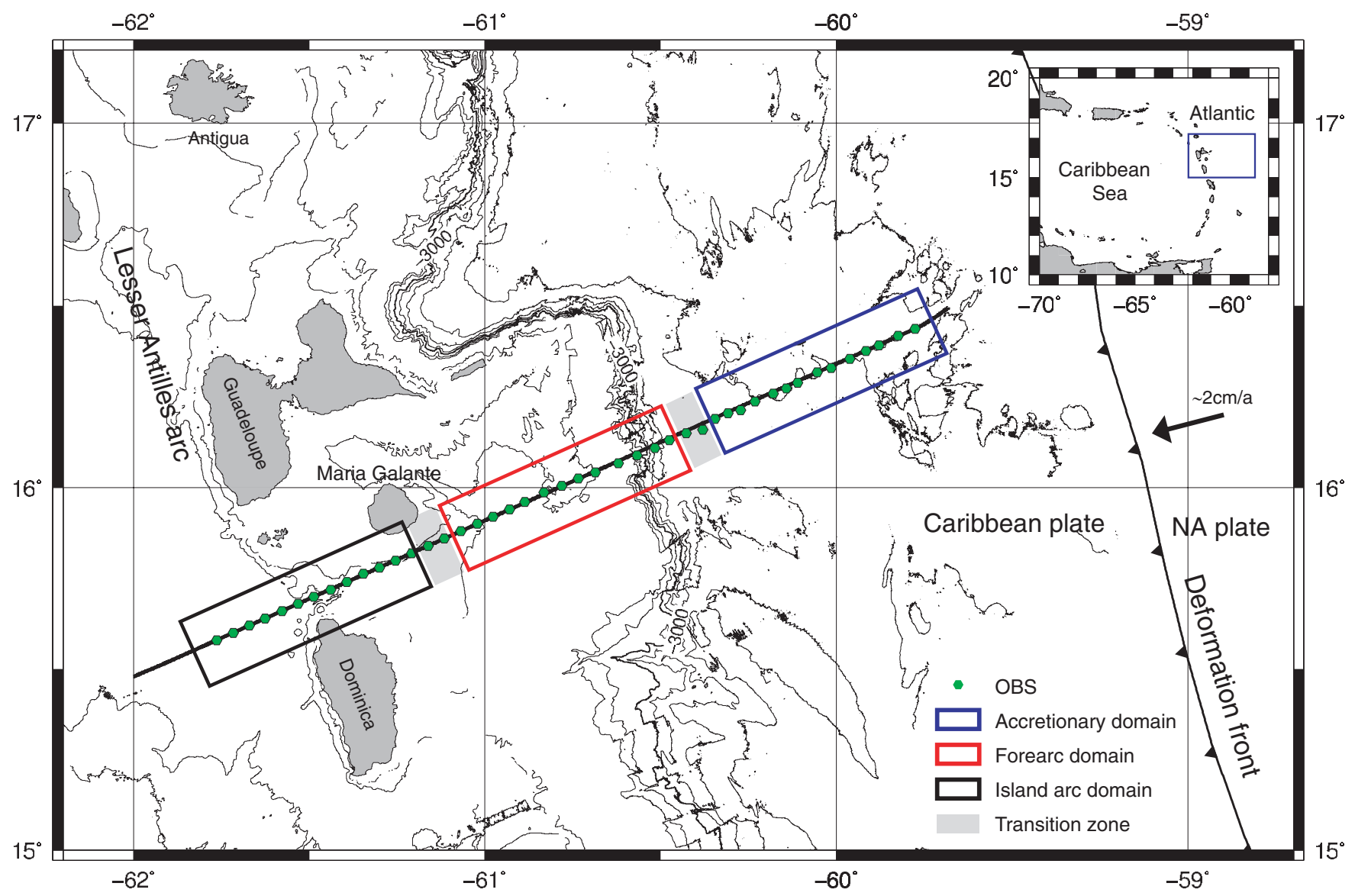

Figure 1. Map of the survey site, showing the deformation front between the North American (NA) and Caribbean plates. Profile P02 of cruise MSM04/2 of the German vessel RV Maria S. Merian was shot at 100-m spacing and a station separation of $5 \mathrm{~km}$. Circles denote relocated positions of the seismic instruments. The color coding of the transect boxes refers to the subsequent data analysis and approximately defines the arc (black), forearc (red), and accretionary (blue) regions. The inset is a regional map of the Lesser Antilles. Bathymetry comes from data sets acquired during cruises EW 9803, MSM 04-2, and Sismantilles I. Convergence rate is from Feuillet (2000). 
estimate of the velocity structure along the transect and provided the basis for defining three distinct geologic parts of the margin: (1) the island arc, (2) the forearc high, and (3) the accretionary complex. The velocity distribution and segment definition agreed with earlier seismic refraction tomography results conducted in the study area (Christeson et al., 2003).

The major drawback of the inversion problem was its nonuniqueness; a family of models could fit the arrivals within the error range. With a total number of $N_{\mathrm{tt}}$ traveltimes with the residuals $\mathbf{d}_{\mathrm{th}}$ as well as the observed residuals $\mathbf{d}_{\mathbf{o b s}}$, a satisfactory model resulted in

$$
\chi^{2}=\frac{\sum_{i=1}^{N_{t t}}\left(\frac{d_{\mathrm{obs}}(i)}{d_{\mathrm{th}}(i)}\right)^{2}}{N_{\mathrm{tt}}}
$$

of nearly one, with an rms misfit close to the assumed picking error. We let $\mathbf{m}$ denote the solution vector of realizations and $E(\mathbf{m})$ be the a posteriori expectation of $\mathbf{m}$ (Tarantola, 1987; Matarese, 1993). With $\sigma_{m}(\mathbf{m})$ being the a posteriori marginal density function, we wrote the a posteriori model covariance matrix $\mathbf{C}$ as

$$
\mathbf{C}=\int[\mathbf{m}-E(\mathbf{m})] \cdot[\mathbf{m}-E(\mathbf{m})]^{T} \sigma_{m}(\mathbf{m}) d \mathbf{m} .
$$

where $T$ denotes transposition. This form of the covariance matrix can be approximated, assuming all realizations $N$ are equally probable. as

$$
\mathbf{C} \approx \frac{1}{N} \sum_{i=1}^{N}\left[\mathbf{m}_{i}-E(\mathbf{m})\right] \cdot\left[\mathbf{m}_{i}-E(\mathbf{m})\right]^{T},
$$

with $\mathbf{m}_{i}$ the $i$ th realization of the solution vector $\mathbf{m}$.

At this stage, we chose a Bayesian approach to identify the resolution of the outcome and to analyze the a posteriori probability density in the model space. This was expressed in form of a product (Tarantola, 1987):

$$
\sigma_{M}(\mathbf{m})=k \rho_{M}(\mathbf{m}) L(\mathbf{m}),
$$

with $k$ an appropriate normalization constant, $\rho_{M}$ the a priori information on the model parameters, and the likelihood function $L(\mathbf{m})$ as a measure of the quality of the model $\mathbf{m}$ in fitting the data (Mosegaard and Tarantola, 1995)

To account for this nonuniqueness, we fitted the velocities of the three distinct regions over a range of $40 \mathrm{~km}$ with Gaussian curves (Figure 2). The regions were expressed in terms of $x_{i}$ :

$$
\begin{aligned}
& x_{1}-20 \leq x_{i} \leq x_{1}+20 \rightarrow \overline{V_{1 k}}, \\
& x_{2}-20 \leq x_{i} \leq x_{2}+20 \rightarrow \overline{V_{2 k}}, \\
& x_{3}-20 \leq x_{i} \leq x_{3}+20 \rightarrow \overline{V_{3 k}},
\end{aligned}
$$

with the corresponding profile $x_{1}=80 \mathrm{~km}, x_{2}=145 \mathrm{~km}$, and $x_{3}$ $=260 \mathrm{~km}$. With equally weighted bins and an (improved) log-likelihood method resulting from the very low statistics $(40 \mathrm{~km} \cong 80$ $x$-nodes) (James and Roos, 1975; Brun and Rademakers, 1997), we were able to use the fitting parameters, mean and deviation, to set up a priori probability densities (see Figure 3 ). Ultimately, they were used to define a model space $M$. Under the assumption of laterally variable transition zones in between the three tectonic regimes, we created more than 50 2D starting models $m\left(V_{1}, V_{2}, V_{3}, z\right) \in M$ for the inversion. A schematic overview of this setup is shown in Figure 4.

The retrieval of the posterior distributions of the input ensemble was achieved in two main steps. Step 1 was prior input. The starting model was defined using velocity distributions $V_{1}(z), V_{2}(z)$, and $V_{3}(z)$, with their corresponding confidence intervals given by the standard deviation. By interpolating linearly in between the two transition zones with variable width, we constructed a 2D initial model for the inversion, which was completely randomized in its corresponding tectonic regions according to its confidence level.

Step 2 was model retrieval. Rather than a forward calculation to retrieve the posterior distibution through a Markov-chain Monte
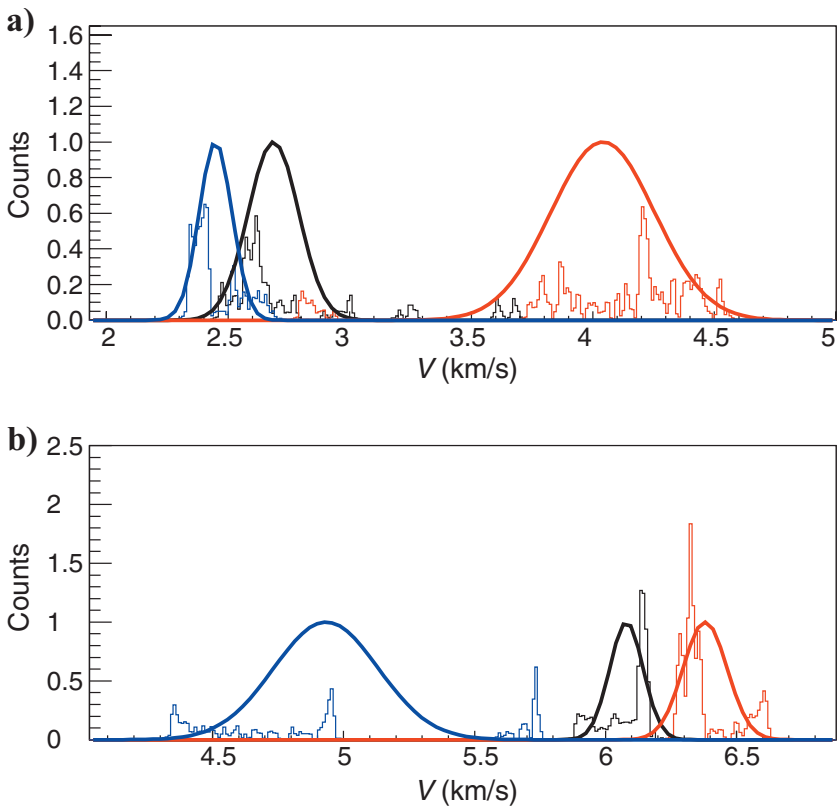

Figure 2. Representative fits of the velocities in the predefined regions used for the Monte Carlo estimation of 2D initial starting models. Fit at (a) $z=1 \mathrm{~km}$ and (b) $z=7 \mathrm{~km}$ below the seafloor (SF). Black curve is the island arc, red curve is the forearc high, and blue curve is the accretionary prism.

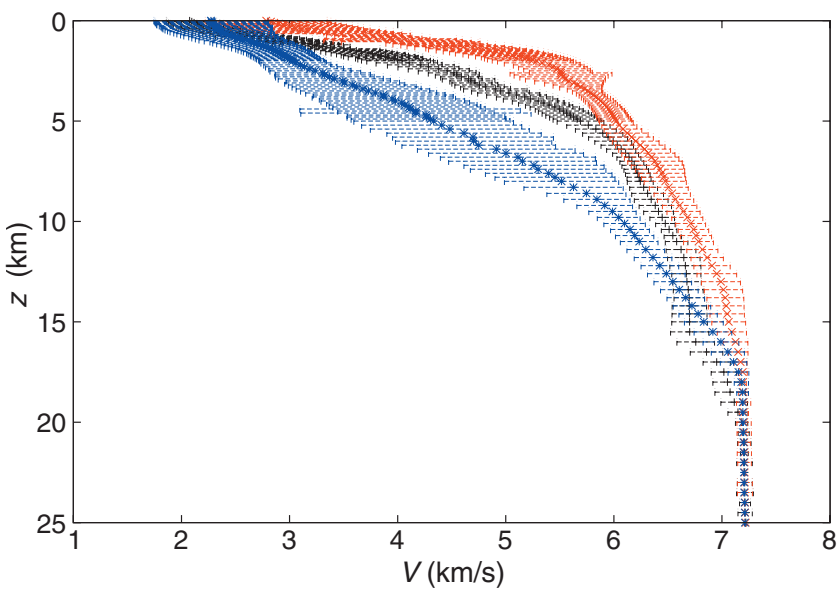

Figure 3 . A priori velocity probability density functions used for creating 2D starting models. Mean values and confidence levels, recovered by the fitting procedure (Figure 2), define the a priori information for parameter estimation. Black markings are the island arc, red are the forearc high, and blue are the accretionary prism. 
Carlo method, we solved the inverse problem (Tarantola, 1987) at hand with the tomographic procedure from Korenaga et al. (2000). To minimize the artifacts introduced by large model updates, we chose a top-to-bottom approach, increasing the number of arrivals according to the offset ranges $0-20 \mathrm{~km}, 0-50 \mathrm{~km}$, and $0-130 \mathrm{~km}$, with spatially varying smoothing and correlation parameters.

\section{RESULTS OF MONTE CARLO ESTIMATION}

The solution of any inverse problem is never one result or image but a probability distribution of samples of the a posteriori probabili-

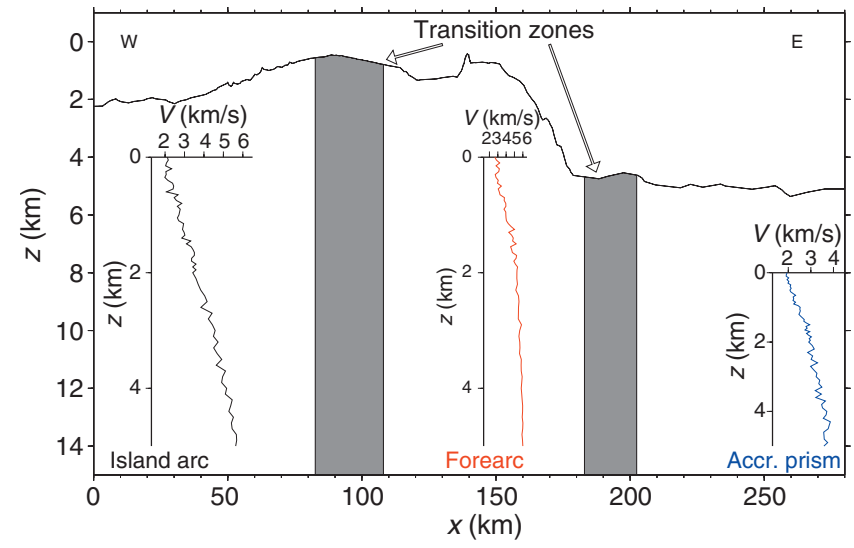

Figure 4. Schematic of model setup along the profile. Representative velocity functions used for the setup of the starting model are depicted; transition zones are dark gray planes in between. Seafloor bathymetry is shown by the slightly thicker black line, based on swath data.

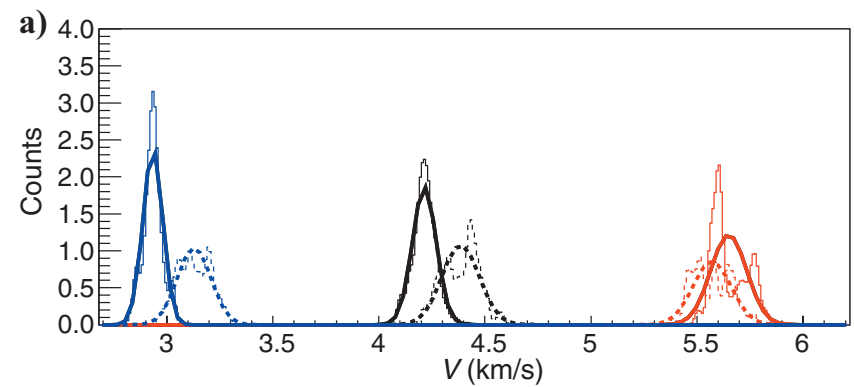

b) 4 .

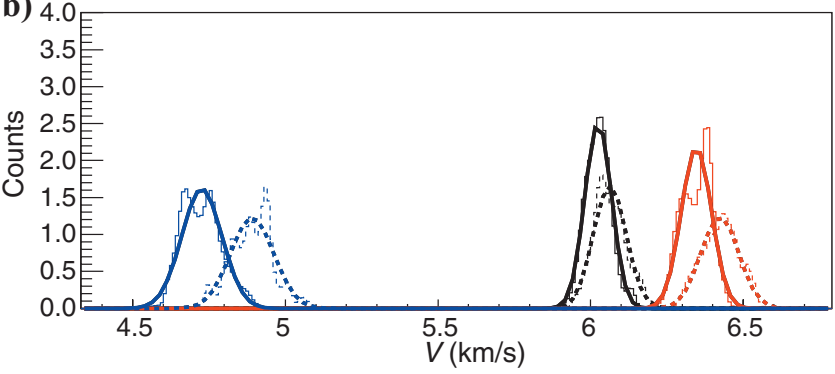

Figure 5. Comparison of a priori probability density (dashed) and a posteriori probability density (solid) at (a) $3.0 \mathrm{~km}$ and (b) $7.2 \mathrm{~km}$ below the seafloor. The histograms were fitted with Gaussian curves to visualize the decrease of the variance, directly visible in the peaks and widths of the distributions, because the amount of statistics is identical. The lateral shift comes from the smoothing of the initial model before the fitting procedure to set up the 2D models. Black curves are the island arc, red curves are the forearc high, and blue curves are the accretionary prism. ty density $\sigma(m)$ (Tarantola, 1987). The practice of calculating the mean of an ensemble of Monte Carlo realizations yields an oversimplified (smoothed) solution to the inverse problem. If a collection of solutions is available, it is possible to give a more quantitative measure for features under investigation rather than merely constraining the interpretation on a smoothed mean.

At this point, we can compare the a priori with the a posteriori probability distributions (Figure 5). The evidence that the P-wave velocities are converging into a minimum can be deduced from the distribution of velocities from the final Monte Carlo ensemble in comparison with the starting models. The variance of the final models is reduced; therefore, velocities converge into a minimum. This test is strongly dependent on ray coverage, as can be seen in the deeper parts of the model. Only where ray coverage is sufficient can we increase the resolution.

The actual power of the statistical approach becomes even more evident if we start analyzing the velocity structure on the basis of our initialization. Calculating the rms values $\Delta V$ at each horizontal grid point $x$ for each model, we are able to analyze the deviations along the profile according to the mean velocity function $\left(\overline{V_{1}}, \overline{V_{2}}, \overline{V_{3}}\right)$ used in the setup:

$$
\Delta V_{i}(x)=\sqrt{\frac{\sum_{k=0}^{n z}\left(\bar{V}_{i}\left(x, z_{k}\right)-V\left(x, z_{k}\right)\right)^{2}}{n z-1}}, \quad i=1,2,3 .
$$

Gaussian fitting of each distribution gives an estimate of the correlation between the mean velocity/depth distributions of the distinct regions and the velocity functions along the profile. The results of this fitting procedure are shown in Figure 6. As expected, the divergence from the mean velocity functions mimics the structural composition of the predefined tectonic regions. The divergence is smallest in the areas of highest resemblance. The transitions between these regions can be quantified by the overlap of distributions moving along the transect (Figure 7). Again, the rms for each region is shown. The superposition of the fits and therefore total distribution is

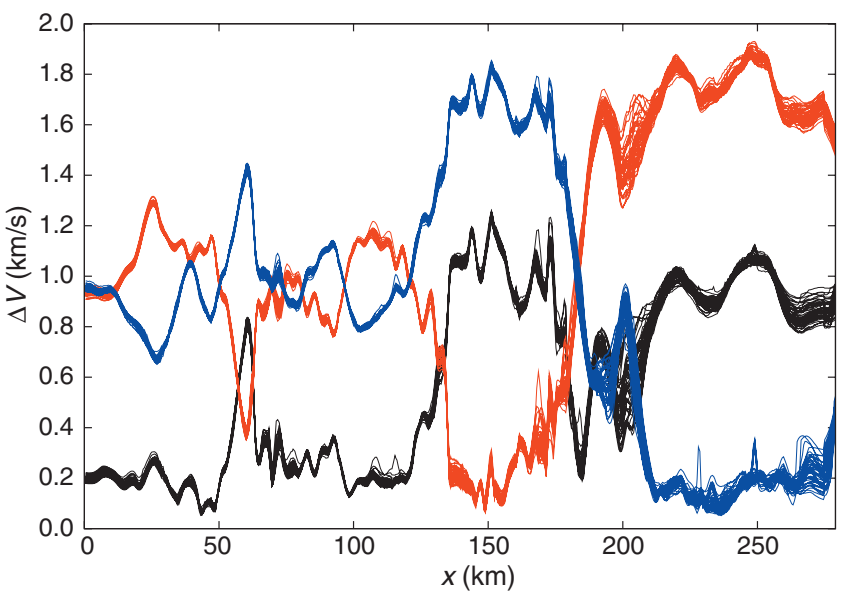

Figure 6. Divergence $\Delta V_{i}$ with $i=1$ (black), $i=2$ (red), and $i=3$ (blue) along the profile. The divergence of each of the models of the resulting Monte Carlo ensemble was calculated according to equation 6. Black curves are the island arc, red curves are the forearc high, and blue curves are the accretionary prism. 
given by

$$
f_{\text {tot }}(x)=\sum_{i=1}^{3} \text { const }_{i} e^{-0.5\left(\left(\overline{d V_{i}}\right) / \sigma_{i}(d V)\right)^{2}},
$$

with const ${ }_{i}, \overline{d V_{i}}$, and $\sigma_{i}(d V)$ the normalization constant, mean, and standard deviation of the misfit to the reference distributions $\bar{V}_{i}$, respectively, and shown by the dashed green line in Figure 7.

The value of equation 6 is in measuring the resemblance or divergence, whereas the value of equation 7 is in measuring the mixing ratio between the given distributions. The validity of dissecting the profile according to significant velocity/depth distributions and comparing the expected distributions with the deduced velocity field can be seen around profile $60 \mathrm{~km}$ (Figure 6). The resemblance to the forearc high function is much higher because we see an increase in seismic velocities close to the seabed.

Between 120 and $140 \mathrm{~km}$, we see a fairly abrupt change in the match to $\Delta V_{1}$ and $\Delta V_{2}$ (Figure 6). This coincides with the transition zone between the forearc high and island arc region. Moving along the transect, the divergence to the forearc high distribution $\Delta V_{2}$ starts increasing from $165 \mathrm{~km}$ until it enters the accretionary complex, where it reaches the highest value of divergence. The distance between 170 and $210 \mathrm{~km}$ is not clearly identifiable as a result of mixing the island arc and accretionary complex distributions $\Delta V_{1}$ and $\Delta V_{3}$ (Figure 6). Between 165 and $190 \mathrm{~km}$, the distributions interchange their divergent behavior.

Besides the small-scale structures mimicked by the match to the mean curves, we can clearly define regions of accretionary, forearc, and island arc character (Figure 6). Moving along the profile, we enter the forearc high at $130 \mathrm{~km}$ and leave it at $180 \mathrm{~km}$, where we enter a sedimentary basin from 180 to $190 \mathrm{~km}$. Strong mixing and a sudden increase in the uncertainty of the fit result indicate a structural change between an island arc and a velocity-field characteristic of a sedimentary accretionary prism. At $202 \mathrm{~km}$, the divergence seems to be large in relation to each fit (Figure 7). One reason for this might be a velocity increase related to a geologic-tectonic backstop feature identified in previous studies (Bangs et al., 2003; Christeson et al., 2003). The parameterization of the model space allows a direct comparison between the velocity structure of a tomographic solution and an assumed velocity distribution for a defined tectonic setting. Furthermore, we can test different tectonic settings given a mean velocity distribution for each of them.

The statistics obtained by variable starting models in a Monte Carlo ensemble help define tolerance intervals for the deviation. We chose two schemes to compare the velocity structure along the profile. The first method allows an overall estimate of conformity $\left(\chi^{2} / \mathrm{rms}\right)$ between the velocity structure at a given position $x$ up to a certain depth in a tomographic solution and the velocity structure we would expect for a predefined tectonic setting. This provides an overall estimate of the divergence along the profile for a tectonic setting ( $x$-estimate). A different approach is a singular comparison of velocity values resulting in a deviation between expected (reference) and calculated (model) values. This yields an estimate of structural change moving deeper in the model and allows a comparison at particular depths ( $x z$-estimate):

$$
\begin{gathered}
\Delta V_{1 k_{\max }}(x)=\sqrt{\frac{\sum_{k=0}^{k_{\max }}\left(\bar{V}_{i}\left(x, z_{k}\right)-V\left(x, z_{k}\right)\right)^{2}}{k_{\max }-1},} \\
1 \leq k_{\max } \leq n z, \\
d V_{1 k}\left(x, z_{k}\right)=\sqrt{\left(\bar{V}_{i}\left(x, z_{k}\right)-V\left(x, z_{k}\right)\right)^{2}} .
\end{gathered}
$$

With these two methods it is possible to analyze the structural changes along the profile. We chose to compare the three tectonic regions used for the Monte Carlo ensemble setup to achieve a higher estimate of equivalence and divergence.

\section{ANALYZING THE BACKSTOP}

The divergence in the transition zone between the forearc and accretionary regions can be better understood by comparing $\Delta V$ of equation 6 - not over the complete range of $\mathrm{z}\left(k=1, \ldots, n_{z}\right)$, but sequentially moving deeper into the model $\left(k=1, \ldots, k_{\max }\right.$; equation $8)$. We would expect a higher resemblance to the accretionary distribution for the shallower terms, i.e., summation up to $k_{\max } \ll n_{z}$, whereas the resemblance to the island arc velocity distribution would become predominant in the deeper part from an increase in velocities. This is visualized in Figure 8 with increasing depth range. Figure 9 shows the distributions at $4.9 \mathrm{~km}$ below the seafloor. If we analyze the mean distributions, adding more values as we move deeper, we see the structural change associated with the three tectonic regimes. This gives a qualitative measure of the velocity structure at a certain $x$-position along the profile and consequently provides a regional approximation of the velocity field.

As a second approach, we chose a direct comparison of velocities at all $z$-node positions along the profile (equation 9; Figure 10). This is a one-bin-thick $z$-filter returning the deviation between the model and expectation values of the mean distributions. We move parallel to the seafloor and calculate the deviation at each position $(x, z)$. The approach allows a direct match between the reference and calculated

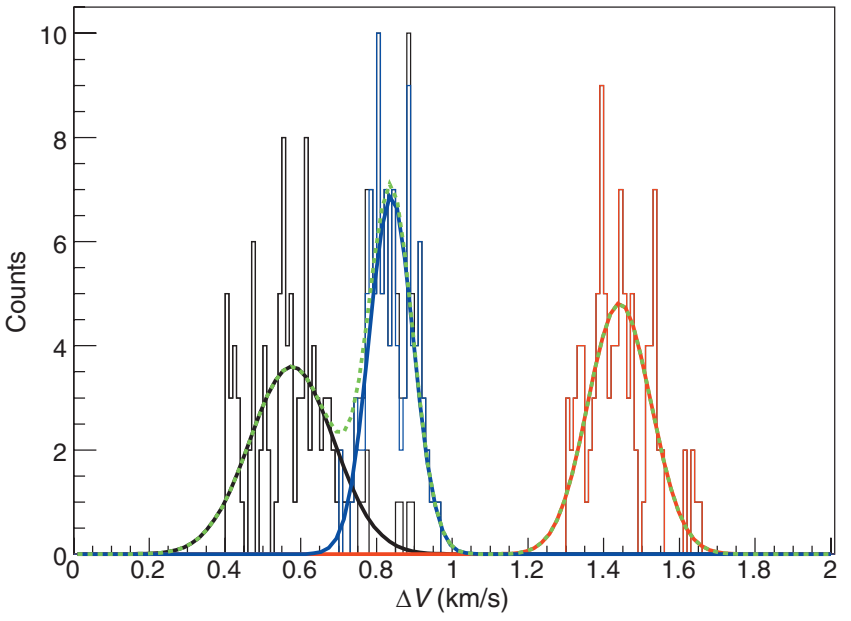

Figure 7. Example of the overlap of the cumulative divergence $\Delta V$ in the transition zone from the forearc high into the accretionary complex. The distributions are projected in the $\Delta V$ plane and subsequently fitted with Gaussian functions. The green curve corresponds to equation 7. Black curve is the island arc, red curve is the forearc high, and blue curve is the accretionary prism. In this figure, $x$ $=201-202 \mathrm{~km}$. 


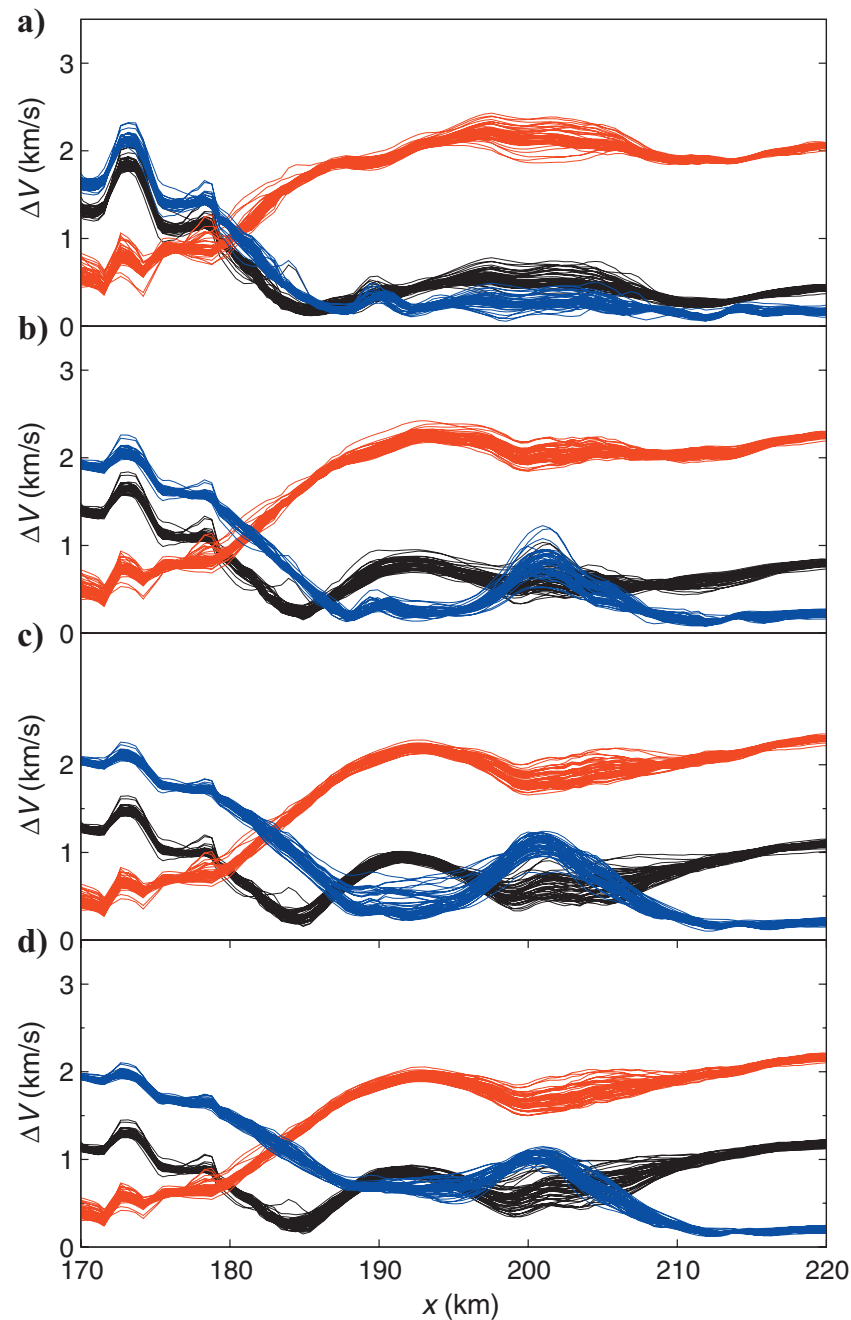

Figure 8. Cumulative divergence of actual $V\left(x_{i}, z_{k}\right)$ to the mean velocity up to depths of $z_{k}$ equals (a) $1.95 \mathrm{~km}$, (b) $3.4 \mathrm{~km}$, (c) $4.9 \mathrm{~km}$, and (d) $9.2 \mathrm{~km}$ below the seafloor between profile $170 \leq x_{i} \leq 220$. Black curves are the island arc, red curves are the forearc high, and blue curves are the accretionary prism.

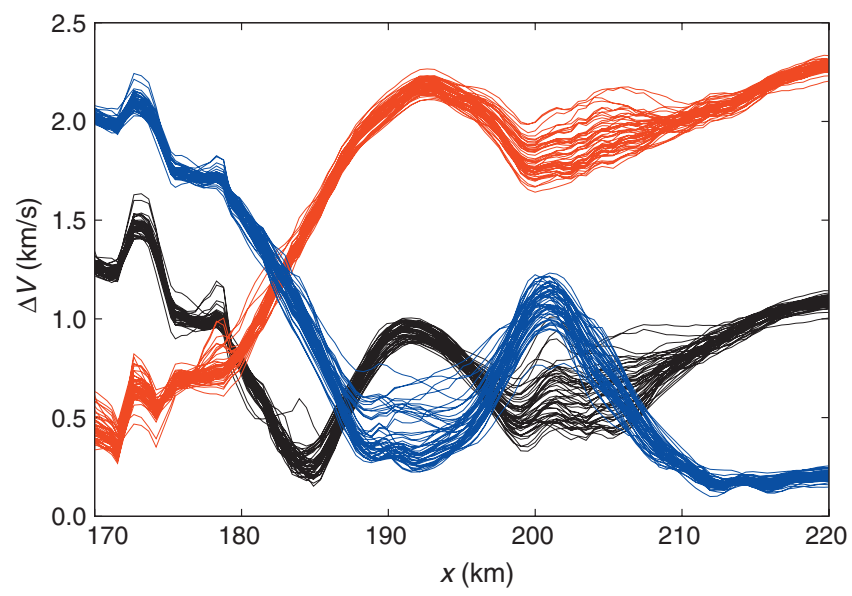

Figure 9. Distribution of $\Delta V$ functions after fit for $z=4.9 \mathrm{~km}\left(k_{\max }\right.$ $=70$ ) below the seafloor, showing a clear distinction in the velocity structure in the vicinity of the backstop feature. Black curves are the island arc, red curves are the forearc high, and blue curves are the accretionary prism. velocities. Because we compare with three different distributions, we have an estimate of the structural setting.

Furthermore, we can provide tolerance intervals of the match to a given distribution by a Gaussian fit at each $x$-position. At profile $195 \mathrm{~km}$, where $z=2.9 \mathrm{~km}$ below the seafloor, the mean velocity of the accretionary distribution at this depth deviates, whereas the mean velocities from the forearc and island arc match. Further tracing the match at depth, the behavior can be mapped (Mosegaard and Tarantola, 1995). This is valid at each $x$-node only where the ray coverage in the model is sufficient. Figure 10 shows the distributions at increasing depths below the seafloor, ultimately yielding a qualitative measure of the velocity structure at a certain position $x$ and a certain depth $z$.

Comparing this with the previous method, we see higher horizontal as well as vertical structural changes in the velocity field and obtain a quantitative measure of divergence for each $(x, z)$ node. The maximum depths reached by the rays in this part of the profile range from 6.5 to $14.5 \mathrm{~km}$ below the seafloor, which makes a comparison

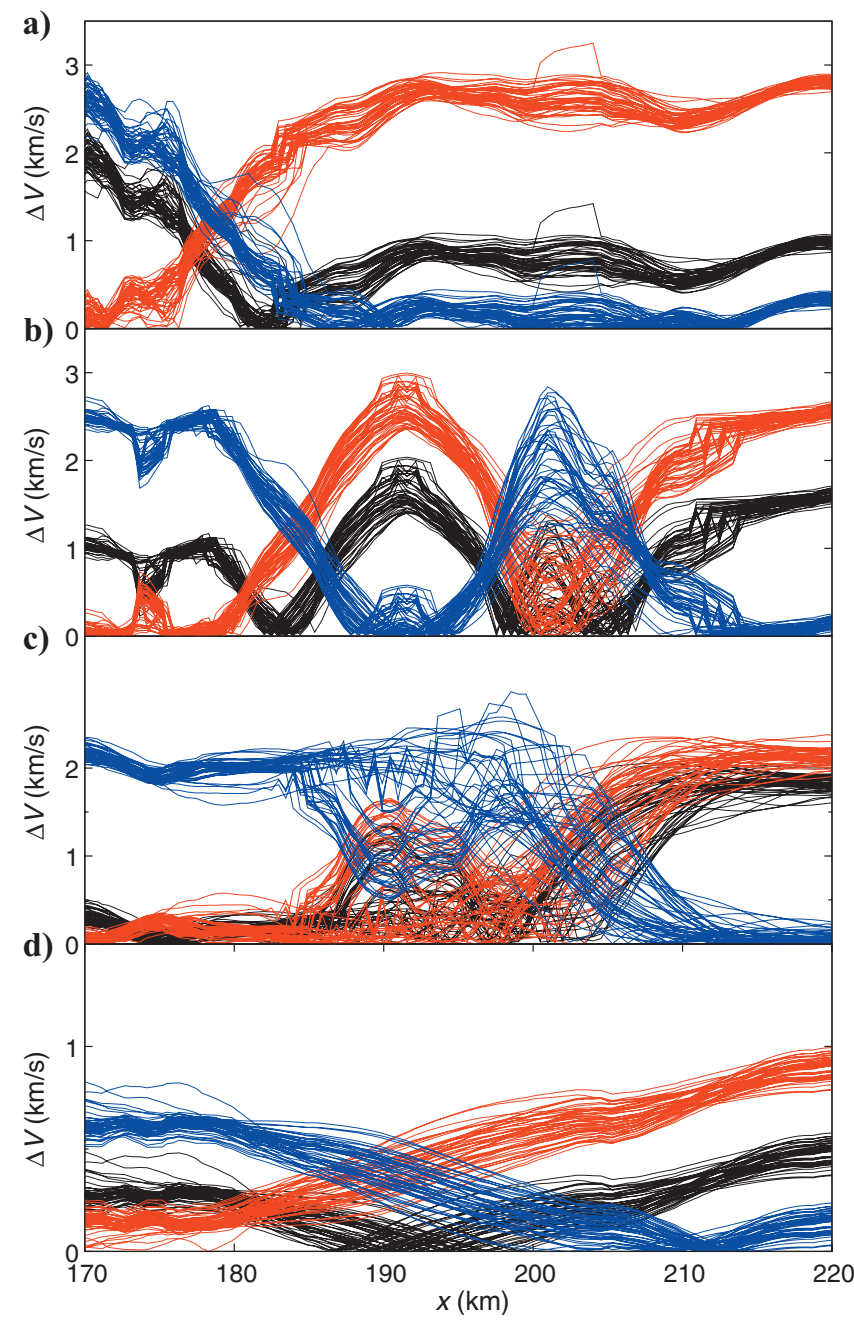

Figure 10. Singular deviation of $V\left(x_{i}, z_{k}\right)$ for $170 \leq x_{i} \leq 220$ to the mean velocity at depths $z_{k}$ equals (a) $1.95 \mathrm{~km}$, (b) $3.4 \mathrm{~km}$, (c) $4.9 \mathrm{~km}$, and (d) $9.2 \mathrm{~km}$ below the seafloor. Clear structural changes are visible below the seafloor. Black curves are the island arc, red curves are the forearc high, and blue curves are the accretionary prism. 


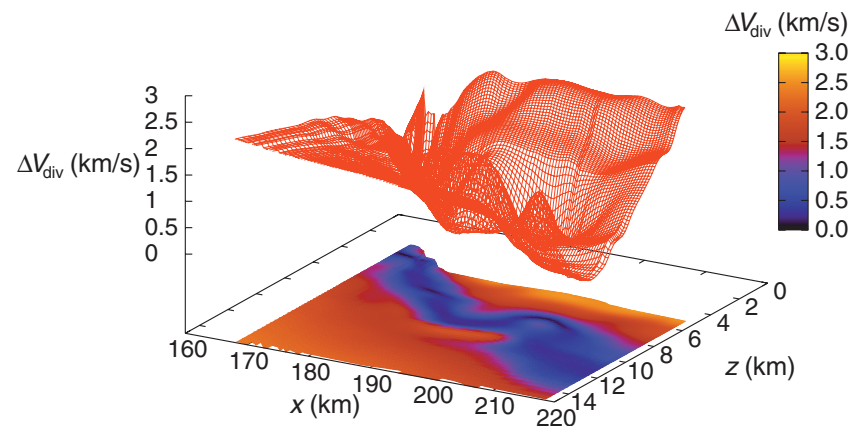

Figure 11. Example of the divergence for one output model moving deeper. The dark areas represent higher matching of the velocity structure in comparison with the mean curve $\overline{V_{2}}$.

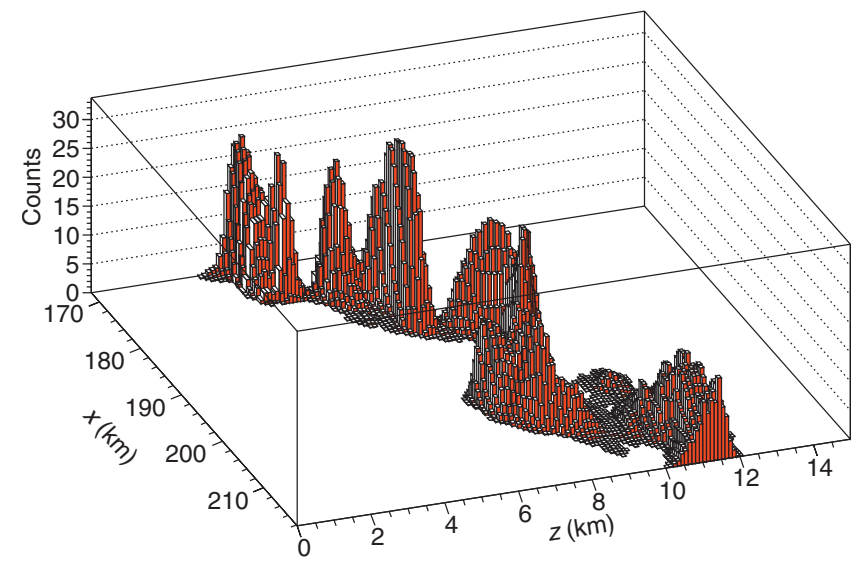

Figure 12. Perspective view of the backstop structure according to a divergence analysis with $15 \leq k_{\mathrm{div}} \leq 25$. Projecting onto the $z$-axis and subsequently fitting the result gives the most probable depths for the backstop along the profile.

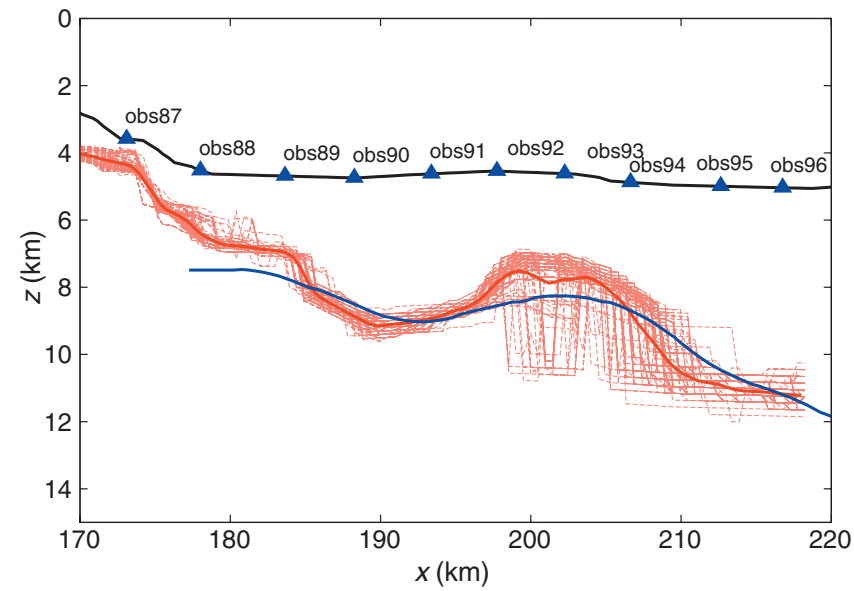

Figure 13. Backstop structure (pastel lines) according to a divergence analysis with $\overline{V_{2}}\left(i, k_{\mathrm{div}}\right) \quad 15 \leq k_{\mathrm{div}} \leq 25$. The parameterization is clearly visible, and the resolution decreases as a result of weaker penetration depths in the accretionary domain. The thick red curve denotes the minimal structure of the backstop; the blue curve denotes the interpretation according to the $4.5-\mathrm{km} / \mathrm{s}$ isocontour line of Christeson et al. (2003); and the black curve is the bathymetry. in deeper parts of the profile impossible, as can be seen in Figure 10d, where no clear match is traceable.

The backstop (Bangs et al., 2003; Christeson et al., 2003) was interpreted by a match between tomographic and near-vertical data analysis. The comparison resulted in a best match of isocontours between 3.5 and $5.5 \mathrm{~km} / \mathrm{s}$. Assuming a geologic relationship between forearc and backstop, we should be able to trace the velocity gradients according to this interpretation. On the basis of the preceding interpretation gained by divergence analysis, we designed a 2D filter. We chose to analyze the $2 \mathrm{D}$ profile with the mean velocity distribution $\overline{V_{2}}$ between $V_{\min }=3.797 \leq V_{\text {div }} \mathrm{km} / s \leq V_{\max }=5.064\left(15 \leq k_{\text {div }}\right.$ $\leq 25)$. Figure 11 shows the divergence of the match moving deeper along the profile for a single model. For each $x$-node, we calculate the divergence $d V$ at each $z$-node.

Doing this for all models, we retrieved a minimal matching distribution of the backstop structure. Figure 12 shows a perspective view of this distribution and visualizes the fitting routine on finding the most probable - i.e., mean — values of the depths according to a best match between forearc and backstop. Projections of the distribution are made in depth and subsequently fitted with Gaussian functions. Figure 13 shows the result of minimal matching of all models. Model binning is clearly visible. We do not reach the same depths in forward-calculating the rays in all of the models, so we can neglect certain results. This analysis is strongly dependent on the information to be gained. However, it generally applies to any facies in a purely homogeneous study if the search algorithm resembles the result.

\section{CONCLUSION}

From this study, we infer several conclusions concerning the interpretation of the tomographic approach. By calculating a global as well as singular misfits of velocities, we are able to map tectonic regions along the profile. Comparing velocity distributions with the result of a tomographic inversion along the profile, we can mimic the subsurface structures in their extent and composition. The possibility of gaining a priori information for seismic refraction analysis by a simple solution to an inverse problem and subsequent resolution of structural heterogeneities through a divergence analysis is a new and simple way of defining a priori space and estimating the a posteriori mean and covariance in singular and general forms.

The major advantage of a Monte Carlo-based approach in our case study is the gained knowledge of velocity/depth distributions. Certainly, the decision of where to extract velocity information on the profile for setting up a Monte Carlo ensemble limits the a priori space. However, the general conclusion of analyzing the velocity field according to distinct reference distributions gives us the possibility of defining the covariance according to any tectonic environment if we have a priori information on the velocity/depth distributions.

Using the wide-angle data recorded across the Lesser Antilles, we resolved a shallow feature like the backstop by a robust and simple divergence analysis. Our new methodology effectively extracts some key features and properties from the inversion results by including information concerning the confidence level of results. This new, simple approach provides a simple solution to an inverse problem and subsequent resolution analysis of structural heterogeneity. 


\section{ACKNOWLEDGMENTS}

We are grateful to the master, officers, and crew of the Maria $S$. Merian cruise 04/ 2 for their cooperation. The refraction data used in this case study were acquired in the scope of the European Unionfunded THALES project (grant FP6-029080-2). We thank C. Papenberg, E. Flueh, D. Klaeschen, A. Krabbenhoeft, A. Hirn, and M. Laigle for many helpful discussions. Thorough reviews by assistant editor M. Sacchi and associate editor B. Wang as well as three anonymous reviewers helped sharpen the manuscript and improved an earlier version.

\section{REFERENCES}

Bangs, N. L., G. L. Christeson, and T. H. Shipley, 2003, Structure of the Lesser Antilles subduction zone backstop and its role in a large accretionary system: Journal of Geophysical Research (Solid Earth), 108, 2358-2372.

Brun, R., and F. Rademakers, 1997, ROOT-An object oriented data analysis framework: Nuclear Instruments and Methods in Physics Research A, 389, 81-86.

Christeson, G. L., N. L. Bangs, and T. H. Shipley, 2003, Deep structure of an island arc backstop, Lesser Antilles subduction zone: Journal of Geophysical Research (Solid Earth), 108, 2327-2343.

Feuillet, N., 2000, Sismotectonique des Petites Antilles: Ph.D. dissertation, Universite Paris VII, Denis Diderot Universite.

Hansen, T. M., A. G. Journel, A. Tarantola, and K. Mosegaard, 2006, Linear inverse Gaussian theory and geostatistics: Geophysics, 71, no. 6, R101R111.

Hjelt, S., 1992, Pragmatic inversion of geophysical data: Springer-Verlag Berlin, Lecture notes in earth sciences 39.
Jacobsen, B. H., K. Mosegaard, and P. Sibani, 1996, Inverse methods: Springer-Verlag Berlin.

James, F., and M. Roos, 1975, Minuit-A system for function minimization and analysis of the parameter errors and correlations: Computer Physics Communications, 10, 343-367.

Kirsch, A., 1996, An introduction to the mathematical theory of inverse problems: Springer-Verlag New York, Inc.

Korenaga, J., W. S. Holbrook, G. M. Kent, P. B. Kelemen, R. S. Detrick, H.-C. Larsen, J. R. Hopper, and T. Dahl-Jensen, 2000, Crustal structure of the southeast Greenland margin from joint refraction and reflection seismic tomography: Journal of Geophysical Research, 105, 21591-21614.

Matarese, J., 1993, Nonlinear traveltime tomography: Ph.D. dissertation, Massachusetts Institute of Technology.

Mosegaard, K., and M. Sambridge, 2002, Topical review: Monte Carlo analysis of inverse problems: Inverse Problems, 18, 29-54.

Mosegaard, K., and A. Tarantola, 1995, Monte Carlo sampling of solutions to inverse problems: Journal of Geophysical Research, 100, 12431-12448.

Parker, R., 1994, Geophysical inverse theory: Princeton University Press.

Parker, R. L., and A. M. Dziewonski, 1995, Geophysical inverse theory: Physics Today, 48, 92 .

Press, F., 1968, Earth models obtained by Monte Carlo inversion: Journal of Geophysical Research, 73, 5223.

1971, An introduction to earth structure and seismotectonics: Proceedings of the International School of Physics Enrico Fermi.

Sambridge, M., and K. Mosegaard, 2002, Monte Carlo methods in geophysical inverse problems: Reviews of Geophysics, 40, 1009-1038.

Sato, T., and B. L. N. Kennett, 2000, Two-dimensional inversion of refraction traveltimes by progressive model development: Geophysical Journal International, 140, 543-558.

Tarantola, A., 1987, Inverse problem theory: Methods for data fitting and model parameter estimation: Elsevier Science Publ. Co., Inc.

Toomey, D. R., S. C. Solomon, and G. M. Purdy, 1994, Tomographic imaging of the shallow crustal structure of the East Pacific rise at 9 deg $30 \mathrm{~min}$ N: Journal of Geophysical Research, 99, 135-159. 